\title{
REDUCTIONS OF PARTIALLY INVARIANT SOLUTIONS OF RANK 1 DEFECT 2 FIVE-DIMENSIONAL OVERALGEBRA OF CONICAL SUBALGEBRA
}

\author{
S.V. KHABIROV
}

\begin{abstract}
Conic flows are the invariant rank 1 solutions of the gas dynamics equations on the three-dimensional subalgebra defined by the rotation operators, translation by time, and uniform dilatation. The generalization of the conic flows are partially invariant solutions of rank 1 defect 2 on the five-dimensional overalgebra of conic subalgebra extended by the operators of space translations noncommuting with rotation. We prove that the extensions of conic flows are reduced either to function-invariant plane stationary solutions or to a double wave of isobaric motions or to the simple wave.
\end{abstract}

Keywords: gas dynamics, conic flows, partially invariant solutions.

\section{INTRODUCTION}

The equations of gas dynamics possesses 11-dimensional Lie algebra of operator. The optimal system of subalgebras was constructed in [1]. A three-dimensional subalgebra in the optimal system with the basis operators $X_{7}=\partial_{\theta}, X_{10}=\partial_{t}, X_{11}=t \partial_{t}+x \partial_{x}+r \partial_{r}$ in the cylindrical coordinate system $(x, r, \theta)$ generates an invariant rank 1 submodel of conic flows [2]. A fifth-dimensional subalgebra has additional operators of space translations along Cartesian coordinates $y, z$,

$$
\begin{aligned}
& X_{2}=\partial_{y}=\cos \theta \partial_{r}-r^{-1} \sin \theta\left(\partial_{\theta}+W \partial_{V}-V \partial_{W}\right), \\
& X_{3}=\partial_{z}=\sin \theta \partial_{r}-r^{-1} \cos \theta\left(\partial_{\theta}+W \partial_{V}-V \partial_{W}\right) .
\end{aligned}
$$

Generalizations of conic flow w.r.t. fifth-dimensional overalgebra are partially partially invariant solutions of rank 1 defect 2 . It is convenient to represent the cylindrical coordinates of the velocity $\vec{u}$ as $U, V=Q \cos \vartheta, W=Q \sin \vartheta(Q \neq 0$, otherwise we deal with the one-dimensional motion). The invariants of the subalgebra are as follows, $U, Q$, the density $\rho$, the entropy $S$, the pressure is determined by the equation of state $p=f(\rho, S)$.

S.V. Khabirov, Reductions of partially invariant Solutions of Rank 1 Defect 2 FiveDIMENSIONAL OVERALGEBRA OF CONICAL SUBALGEBRA.

(C) Khabirov S.V. 2013.

The work is supported by RFBR (grants 11-01-00026-a, 11-01-00047-a), the Grant of the President of Russia for supporting leading scientific schools (no. NSh-2826.2008.1), the grant No.11.G34.31.0042 of the government of Russia under the decree no. 220.

Submitted January 10, 2012. 
The equations of gas dynamics in the mentioned variables cast into the form

$$
\begin{aligned}
& U_{t}+U U_{x}+Q\left(U_{r} \cos \vartheta+r^{-1} U_{\theta} \sin \vartheta\right)+\rho^{-1} p_{x}=0, \\
& Q_{t}+U Q_{x}+Q\left(Q_{r} \cos \vartheta+r^{-1} Q_{\theta} \sin \vartheta\right)+\rho^{-1}\left(p_{r} \cos \vartheta+r^{-1} p_{\theta} \sin \vartheta\right)=0, \\
& \vartheta_{t}+U \vartheta_{x}+Q\left(\vartheta_{r} \cos \vartheta+r^{-1}\left(\vartheta_{\theta}+1\right) \sin \vartheta\right)+ \\
& \quad+\rho^{-1} Q^{-1}\left(-p_{r} \sin \vartheta+r^{-1} p_{\theta} \cos \vartheta\right)=0, \\
& \rho_{t}+U \rho_{x}+Q\left(\rho_{r} \cos \vartheta+r^{-1} \rho_{\theta} \sin \vartheta\right)+ \\
& \quad+\rho\left[U_{x}+Q_{r} \cos \vartheta+r^{-1} Q_{\theta} \sin \vartheta+Q\left(-\vartheta_{r} \sin \vartheta+r^{-1}\left(\vartheta_{\theta}+1\right) \cos \vartheta\right)\right]=0, \\
& S_{t}+U S_{x}+Q\left(S_{r} \cos \vartheta+r^{-1} S_{\theta} \sin \vartheta\right)=0 .
\end{aligned}
$$

The representation of partially invariant solution of rank 1 defect 2 is as follows; the functions $U, Q, \rho, S, p$ depend on one non-constant parameter $\alpha$, the functions $\alpha, \vartheta$ are generic, i.e., depend on $t, x, r, \theta$.

The substitution of the representation for the solution into the equations of gas dynamics yields an overdetermined system of equations (the main equations of submodel),

$$
\begin{aligned}
& S_{\alpha} Y \alpha=0, \quad U_{\alpha} Y \alpha+\rho^{-1} p_{\alpha} \alpha_{x}=0 \\
& Q_{\alpha} Y \alpha+\rho^{-1} p_{\alpha}\left(\alpha_{r} \cos \vartheta+r^{-1} \alpha_{\theta} \sin \vartheta\right)=0 \\
& \rho^{-1} \rho_{\alpha} Y \alpha+U_{\alpha} \alpha_{x}+Q_{\alpha}\left(\alpha_{r} \cos \vartheta+r^{-1} \alpha_{\theta} \sin \vartheta\right)+ \\
& +Q\left(-\vartheta_{r} \sin \vartheta+r^{-1}\left(\vartheta_{\theta}+1\right) \cos \vartheta\right)=0, \\
& \vartheta_{t}+U \vartheta_{x}+Q\left(\vartheta_{r} \cos \vartheta+r^{-1}\left(\vartheta_{\theta}+1\right) \sin \vartheta\right)+ \\
& \quad+\rho^{-1} Q^{-1} p_{\alpha}\left(-\alpha_{r} \sin \vartheta+r^{-1} \alpha_{\theta} \cos \vartheta\right)=0,
\end{aligned}
$$

where $Y \alpha=\alpha_{t}+U \alpha_{x}+Q\left(\alpha_{r} \cos \vartheta+r^{-1} \alpha_{\theta} \sin \vartheta\right)$.

\section{NON-ISENTROPIC MOTION}

If $S_{\alpha} \neq 0$, the main equations of the submodel become

$$
\begin{aligned}
Y \alpha=0, \quad p_{\alpha} \alpha_{x}=0, \quad p_{\alpha}\left(\alpha_{r} \cos \vartheta+r^{-1} \alpha_{\theta} \sin \vartheta\right)=0, \\
U_{\alpha} \alpha_{x}+Q_{\alpha}\left(\alpha_{r} \cos \vartheta+r^{-1} \alpha_{\theta} \sin \vartheta\right)+ \\
+Q\left(-\vartheta_{r} \sin \vartheta+r^{-1}\left(\vartheta_{\theta}+1\right) \cos \vartheta\right)=0, \\
\vartheta_{t}+U \vartheta_{x}+Q\left(\vartheta_{r} \cos \vartheta+r^{-1}\left(\vartheta_{\theta}+1\right) \sin \vartheta\right)+ \\
+\rho^{-1} Q^{-1} p_{\alpha}\left(-\alpha_{r} \sin \vartheta+r^{-1} \alpha_{\theta} \cos \vartheta\right)=0 .
\end{aligned}
$$

1.1. Non-isobaric motion. If $p_{\alpha} \neq 0$, it follows from (1.1) that

$$
\begin{gathered}
\alpha_{t}=\alpha_{x}=0, \quad \alpha_{r} \cos \vartheta+r^{-1} \alpha_{\theta} \sin \vartheta=0 \Rightarrow \\
\left(-\alpha_{r} \sin \vartheta+r^{-1} \alpha_{\theta} \cos \vartheta\right) \vartheta_{\lambda}=0, \quad \lambda=t, x .
\end{gathered}
$$


It implies $\vartheta_{t}=\vartheta_{x}=0$, since the function $\alpha$ is non-constant. There happens a reduction to a plane stationary solution which an invariant solution on a subalgebra $\left\{\partial_{t}, \partial_{x}\right\}$. The system (1.1) reduces to three equations

$$
\vartheta_{\theta}+1=\operatorname{tg} \vartheta \vartheta_{\tau}, \quad n_{\tau}=\operatorname{tg} \vartheta \vartheta_{\tau}, \quad n_{\tau}+\operatorname{tg} \vartheta n_{\theta}=0,
$$

where $n(\alpha)=\int p_{\alpha} \rho^{-1} Q^{-2} d \alpha, \tau=\ln r$. One of the equation is integrable

$$
n=-\ln |\cos \vartheta|+k(\theta),
$$

other two cast into the form

$$
\cos ^{-2} \vartheta \vartheta_{\tau}=-k^{\prime}+\operatorname{tg} \vartheta, \quad \cos ^{-2} \vartheta \vartheta_{\theta}=-1-\operatorname{tg} \vartheta k^{\prime} .
$$

The compatibility conditions give the equation for the function $k(\theta), k^{\prime \prime}+k^{\prime 2}+1=0$, whose solution $k=\ln |\cos \theta|+k_{0}$ is defined up to the translation w.r.t. $\theta$ admitted by system (1.1), $k_{0}$ is a constant.

Integration of system (1.2) gives a family of functionally-invariant solutions

$$
\operatorname{tg} \vartheta+\operatorname{tg} \theta=\mu_{0} r \cos ^{-1} \theta, \quad n(\alpha)=k_{0}+\ln \left|\frac{\cos \theta}{\cos \vartheta}\right|
$$

depending on two constants $\mu_{0}, k_{0}$ and three arbitrary functions $S(\alpha), \rho(\alpha), Q(\alpha)$.

1.2. Isobaric motion. Let $p_{\alpha}=0$, i.e., $f(\rho, S)=p_{0}$ is constant. Then system (1.1) becomes

$$
\begin{aligned}
& \alpha_{t}+U \alpha_{x}+Q\left(\alpha_{r} \cos \vartheta+r^{-1} \alpha_{\theta} \sin \vartheta\right)=0, \\
& \vartheta_{t}+U \vartheta_{x}+Q\left(\vartheta_{r} \cos \vartheta+r^{-1}\left(\vartheta_{\theta}+1\right) \sin \vartheta\right)=0, \\
& U \alpha_{x}+Q_{\alpha}\left(\alpha_{r} \cos \vartheta+r^{-1} \alpha_{\theta} \sin \vartheta\right)+ \\
& \quad+Q\left(-\vartheta_{r} \sin \vartheta+r^{-1}\left(\vartheta_{\theta}+1\right) \cos \vartheta\right)=0 .
\end{aligned}
$$

The latter equations is equivalent to

$$
\operatorname{div} \vec{u}=0 .
$$

It is convenient to deal with Lagrange variables

$$
\begin{gathered}
\frac{d x}{d t}=U(\alpha), \quad \frac{d r}{d t}=Q(\alpha) \cos \vartheta, \quad r \frac{d \theta}{d t}=Q(\alpha) \sin \vartheta, \\
\left.x\right|_{t=0}=x_{0},\left.\quad r\right|_{t=0}=r_{0},\left.\quad \theta\right|_{t=0}=\theta_{0} .
\end{gathered}
$$

Each solution to system 1 can be written by Cauchy problem 1.5 as $\alpha(t, x, r, \theta)=$ $\beta\left(t, x_{0}, r_{0}, \theta_{0}\right), \vartheta(t, x, r, \theta)=\sigma\left(t, x_{0}, r_{0}, \theta_{0}\right)$.

By (1.3) we have the identities

$$
\beta_{t}=0, \quad \sigma_{t}+\theta_{t}=0 \Rightarrow \beta=\beta\left(x_{0}, r_{0}, \theta_{0}\right), \quad \sigma+\theta=\gamma\left(x_{0}, r_{0}, \theta_{0}\right) .
$$

Due to these identities the solution to problem (1.5) reads as

$$
x=U(\beta) t+x_{0}, \quad r \cos (\gamma-\theta)=Q(\beta) t \cos \left(\gamma-\theta_{0}\right), \quad r \sin (\gamma-\theta)=r_{0} \sin \left(\gamma-\theta_{0}\right) .
$$

In Cartesian coordinates two latter identities are written as

$$
y=r \cos \theta=Q(\beta) t \cos \gamma+y_{0}, \quad z=r \sin \theta=Q(\beta) t \sin \gamma+z_{0},
$$

where $y_{0}=r_{0} \cos \theta_{0}, z_{0}=r_{0} \sin \theta_{0}$. 
Thus, the world line are straight ones. The velocities in Cartesian coordinates are represented by the formulae

$$
\begin{gathered}
u=U(\beta)=u_{0}, \\
v=V \cos \theta-W \sin \theta=Q(\beta) \cos \gamma=v_{0}, \\
w=V \sin \theta+W \cos \theta=Q(\beta) \sin \gamma=w_{0} .
\end{gathered}
$$

By Euler formula $J_{t}=J \operatorname{div} \vec{u}$ and identity (1.4), the Jacobian of the passage from Lagrange coordinates to Euler ones equals one $J=1$ or by (1.6), (1.7)

$$
1=\left|I+t \frac{\partial \vec{u}_{0}}{\partial \vec{x}_{0}}\right|
$$

where $I$ is the unit matrix, $\partial \vec{u}_{0} / \partial \vec{x}_{0}$ is the matrix of partial derivatives, the variable $t$ is free.

It implies that all the invariants of the matrix $\partial \vec{u}_{0} / \partial \vec{x}_{0}$ vanish,

$$
\begin{gathered}
u_{0 x_{0}}+v_{0 y_{0}}+w_{0 z_{0}}=0 \\
\left|\begin{array}{cc}
u_{0 x_{0}} & u_{0 y_{0}} \\
v_{0 x_{0}} & v_{0 y_{0}}
\end{array}\right|+\left|\begin{array}{cc}
u_{0 x_{0}} & u_{0 z_{0}} \\
w_{0 x_{0}} & w_{0 z_{0}}
\end{array}\right|+\left|\begin{array}{cc}
v_{0 y_{0}} & v_{0 z_{0}} \\
w_{0 y_{0}} & w_{0 z_{0}}
\end{array}\right|=0, \\
\operatorname{det} \frac{\partial \vec{u}_{0}}{\partial \vec{x}_{0}}=0 .
\end{gathered}
$$

The general solution to this system was obtained in [3]. In our case we obtain particular solutions, namely, the solutions of a double wave type,

$$
(\vec{a} \cdot \nabla \beta)(\vec{b} \cdot \nabla \gamma)=(\vec{b} \cdot \nabla \beta)(\vec{a} \cdot \nabla \gamma), \quad \vec{a} \cdot \nabla \beta=\vec{b} \cdot \nabla \gamma
$$

where $\vec{a}=\left(U^{\prime}, Q^{\prime} \cos \gamma, Q^{\prime} \sin \gamma\right), \vec{b}=(0,-Q \sin \gamma, Q \cos \gamma), \vec{a} \cdot \vec{b}=0$. As it follows from [3], the level lines of the double wave are second order planar curves.

\section{ISENTROPIC MOTION}

Let $S=S_{0}$ be constant. Then the main equations can be written as

$$
\begin{gathered}
\vartheta_{t}+U \vartheta_{x}+Q\left(\vartheta_{r} \cos \vartheta+r^{-1}\left(\vartheta_{\theta}+1\right) \sin \vartheta\right)+ \\
+\rho^{-1} Q^{-1} p^{\prime}\left(-\alpha_{r} \sin \vartheta+r^{-1} \alpha_{\theta} \cos \vartheta\right)=0 \\
-\vartheta_{r} \sin \vartheta+r^{-1}\left(\vartheta_{\theta}+1\right) \cos \vartheta=c(\alpha) \alpha_{x}, \\
\alpha_{r} \cos \vartheta+r^{-1} \alpha_{\theta} \sin \vartheta=Q^{\prime} U^{\prime-1} \alpha_{x}, \\
\alpha_{t}+b(\alpha) \alpha_{x}=0
\end{gathered}
$$

where $b(\alpha)=U+\rho^{-1} U^{\prime-1} p^{\prime}+Q Q^{\prime} U^{\prime-1}, c(\alpha)=\rho^{-2} Q^{-1} U^{\prime-1}\left(p^{\prime} \rho^{\prime}-\rho^{2}\left(U^{\prime 2}+Q^{\prime 2}\right)\right)$.

The general solution to equation (2.4) can be written implicitly as

$$
x-b(\alpha) t=g(\alpha, r, \theta),
$$

where $g$ is an arbitrary function. We introduce new independent variables $\alpha, r, \theta, x$. The derivatives w.r.t. old variables are expressed in terms of the derivatives of the functions $g$ and $\bar{\vartheta}(\alpha, t, r, \theta)=\vartheta(t, x, r, \theta)$ by the formulae

$$
\begin{gathered}
\alpha_{t}=-\frac{b}{g_{\alpha}+t b^{\prime}}, \quad \alpha_{x}=\frac{1}{g_{\alpha}+t b^{\prime}}, \quad \alpha_{r}=-\frac{g_{r}}{g_{\alpha}+t b^{\prime}}, \quad \alpha_{\theta}=-\frac{g_{\theta}}{g_{\alpha}+t b^{\prime}} ; \\
\vartheta_{x}=\bar{\vartheta}_{\alpha} \alpha_{x}, \quad \vartheta_{\lambda}=\bar{\vartheta}_{\lambda}+\bar{\vartheta}_{\alpha} \alpha_{\lambda}, \quad \lambda=t, r, \theta,
\end{gathered}
$$

where $t=(x-g) b^{-1}$. 
Equation $(2.3)$ becomes

It yields $\bar{\vartheta}_{t}=0$.

$$
g_{r} \cos \bar{\vartheta}+r^{-1} g_{\theta} \sin \bar{\vartheta}=-Q^{\prime} U^{\prime-1}
$$

Equation $(2.2)$ in new variables

$$
\left[-\bar{\vartheta}_{r}\left(g_{\alpha}+t b^{\prime}+\bar{v}_{\alpha} g_{r}\right)\right] \sin \bar{\vartheta}+r^{-1} \cos \bar{\vartheta}\left[\left(\bar{\vartheta}_{\theta}+1\right)\left(g_{\alpha}+t b^{\prime}\right)-g_{\theta} \bar{\vartheta}_{\alpha}\right]=c(\alpha)
$$

involves a free variable $t$. Equating the coefficient at the free variable to zero implies the identities

$$
\begin{gathered}
-\bar{\vartheta}_{r} \sin \bar{\vartheta}+r^{-1}\left(\bar{\vartheta}_{\theta}+1\right) \cos \bar{\vartheta}=0, \\
g_{r} \sin \bar{\vartheta}-r^{-1} g_{\theta} \cos \bar{\vartheta}=c(\alpha) \bar{\vartheta}_{\alpha}^{-1} .
\end{gathered}
$$

Similar procedure for equation (2.1) yield the identities

$$
\begin{gathered}
\bar{\vartheta}_{r} \cos \bar{\vartheta}+r^{-1}\left(\bar{\vartheta}_{\theta}+1\right) \sin \bar{\vartheta}=0 \\
\bar{\vartheta}_{\alpha}=k^{\prime}(\alpha)=\left(\frac{c p^{\prime} U^{\prime}}{\rho Q\left(b U^{\prime}-U U^{\prime}-Q Q^{\prime}\right)}\right)^{1 / 2} .
\end{gathered}
$$

It follows from identities (2.7), 2.9$),(2.10)$ that $\bar{\vartheta}_{r}=0, \bar{\vartheta}_{\theta}=-1 \Rightarrow \bar{\vartheta}=k(\alpha)-\theta$. In view of the obtained identity, equations (2.8), (2.6) can be integrated

$$
g=h(\alpha)+r\left(c k^{\prime-1} \sin (k-\theta)-Q^{\prime} U^{\prime-1} \cos (k-\theta)\right),
$$

and general solution (2.6) casts into the form

$$
x-b(\alpha) t=h(\alpha)+y\left(c k^{\prime-1} \sin k-Q^{\prime} U^{\prime-1} \cos k\right)+z\left(-c k^{\prime-1} \cos k-Q^{\prime} U^{\prime-1} \sin k\right) .
$$

It follows that level surface ( $\alpha=$ const) is a plane as for the simple wave [2].

\section{BIBLIOGRAPHY}

1. L.V. Ovsyannikov. The "PODMODELI" program. Gas dynamics // Prikl. matem. i mekh. 1994. V. 58, No. 4. P. 30-55. [J. Appl. Math. Mech. 1994. V. 58, No. 4. P. 601-627.]

2. S.V. Khabirov. Analytic methods in gas dynamics. Gilem, Ufa. 2003. (in Russian).

3. L.V. Ovsyannikov. Isobaric gas motions // Diff. uranv. 1994. V. 30, No. 10. P. 1792-1799. [Diff. Eqs. 1994. V. 30, No. 10. P. 1656-1662.]

Salavat Valeevich Khabirov, Institute of Mechanics USC RAS,

Oktyabr' av., 71, 450054, Ufa, Russia

E-mail: habirov@anrb.ru 